\title{
Asymptotic Scaling and Monte Carlo Data
}

\author{
A. Trivini* \\ University of Wales Swansea \\ E-mail: pyateswan.ac.uk \\ C. R. Allton \\ University of Wales Swansea \\ E-mail: c.alltoneswansea.ac.uk
}

It is a generally known problem that the behaviour predicted from perturbation theory for asymptotically free theories like QCD, i.e. asymptotic scaling, has not been observed in Monte Carlo simulations when the series is expressed in terms of the bare coupling $g_{0}$. This discrepancy has been explained in the past with the poor convergence properties of the perturbative series in the $g_{0}$. An alternative point of view, called Lattice-Distorted Perturbation Theory proposes that lattice artifacts due to the finiteness of the lattice spacing $a$ cause the disagreement between Monte Carlo data and perturbative scaling. Following this alternative scenario, we fit recent quenched data from different observables to fitting functions that include these cut-off effects, confirming that the lattice data are well reproduced by $g_{0}$-PT with the simple addition of terms $\mathscr{O}\left(a^{n}\right)$.

XXIIIrd International Symposium on Lattice Field Theory

25-30 July 2005

Trinity College, Dublin, Ireland

${ }^{*}$ Speaker. 


\section{Asymptotic scaling}

The so-called $\beta$-function quantifi es the dependence of the coupling constant on the lattice spacing $a$ :

$$
\beta\left(g^{2}\right)=-a \frac{d g^{2}}{d a} .
$$

Integrating this expression one obtains a relation between $g$ and $a$ which is the usual expression for the running of the coupling:

$$
a^{-1}\left(g^{2}\right)=\frac{\Lambda}{f_{P T}\left(g^{2}\right)}
$$

where

$$
f_{P T}\left(g^{2}\right)=e^{-\frac{1}{2 b_{0} g^{2}}}\left(b_{0} g^{2}\right)^{\frac{-b_{1}}{2 b_{0}^{2}}}\left(1+d_{2} g^{2}\right)
$$

is the 3-loop scaling function, $b_{0}, b_{1}$ and $d_{2}$ the usual 1,2 and 3-loop coeffi cients, and $\Lambda$ is a constant of integration. For any lattice prediction of QCD to have physical relevance it should follow the asymptotic scaling according to Eqs. (1.2) and (1.3), in the limit of the bare coupling $g_{0} \rightarrow 0$.

Figure 1 is an example of the strong disagreement between Monte Carlo data and $g_{0}-\mathrm{PT}$, where the data is taken from [1]. ${ }^{1}$ We fi t the same set of data in different renormalized schemes known in the literature; the $g_{V}$ schemes were introduced in [2], the $g_{E}$ in [3], and the $g_{E 2}$ in [4]. We can see that the mismatch decreases using a renormalized coupling constant instead of the bare one [2].

However, a better reduction (see Fig. 1) is obtained with Lattice-Distorted PT [5], using $g_{0}$ as expansion parameter and including lattice artifacts $\mathscr{O}\left(a^{n}\right)$ due to the systematic error in the Monte Carlo data due to the fi niteness of $a$. The expression (1.2) becomes:

$$
a_{L}^{-1}\left(g_{0}^{2}\right)=\frac{\Lambda_{L}}{f_{P T}\left(g_{0}^{2}\right)} \times\left(1+\sum_{n=1} c_{n}^{\prime}\left(g_{0}^{2}\right) f_{P T}^{n}\left(g_{0}^{2}\right)\right)
$$

where $\Lambda_{L}$ is the scale parameter of lattice QCD.

\section{Lattice-Distorted PT}

Eq. (1.4) can be written in a more useful form by including the leading $\mathscr{O}\left(a^{n}\right)$ and next-toleading $\mathscr{O}\left(a^{n^{\prime}}\right)$ terms only: :

$$
a_{L}^{-1}\left(g_{0}^{2}\right)=\frac{\Lambda_{L}}{f_{P T}\left(g_{0}^{2}\right)} \times\left[1-X_{n, v} \frac{g_{0}^{v} f_{P T}^{n}\left(g_{0}^{2}\right)}{G_{0}^{v} f_{P T}^{n}\left(G_{0}^{2}\right)}-Y_{n^{\prime}, v^{\prime}} \frac{g_{0}^{v^{\prime}} f_{P T}^{n^{\prime}}\left(g_{0}^{2}\right)}{G_{0}^{v^{\prime}} f_{P T}^{n^{\prime}}\left(G_{0}^{2}\right)}\right],
$$

where $G_{0}$ is some convenient, reference value of $g_{0}$, e.g. we take $G_{0}=1$ for the Wilson data (corresponding to $\beta=6$ ). In Eq. (1.4), the values of $n, n^{\prime}, v$ and $v^{\prime 2}$ depend on the lattice action

\footnotetext{
${ }^{1}$ The lattice spacing in this fi gure, $a_{r_{c}}$ is introduced in [1] and defi ned via the force, $F(r)$, in an analogous fashion to $r_{0}$, but with $r_{c}^{2} F_{c}\left(r_{c}\right)=0.65$.

${ }^{2}$ Normally $v \equiv v^{\prime}$.
} 


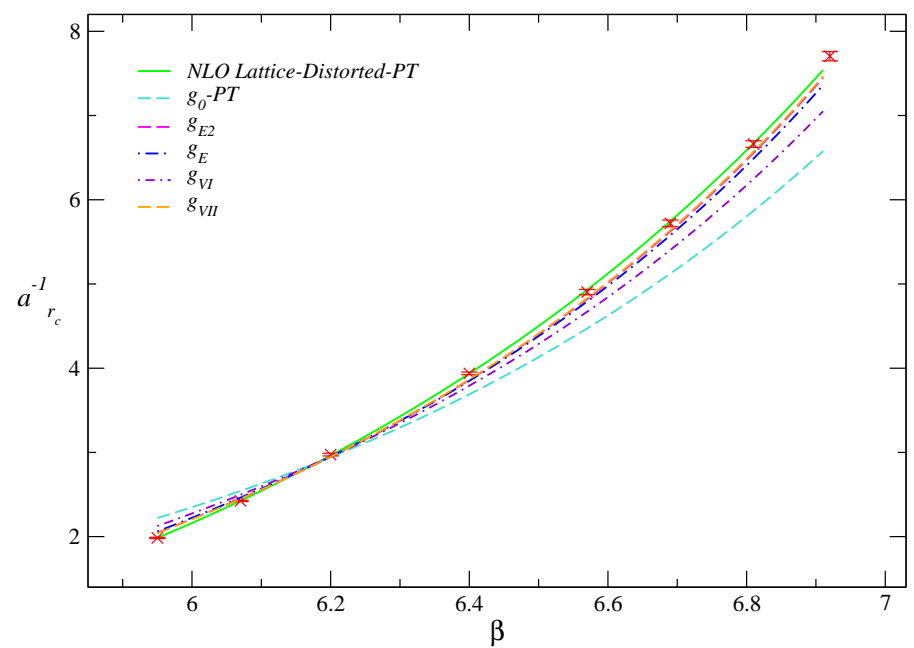

Figure 1: Plot of data points $a^{-1}$ obtained from $r_{c}$ with the Wilson action together with fits from next-toleading order lattice-distorted PT and from PT in different renormalised schemes (see text). The data was taken from [1].

\begin{tabular}{|ccc|llll|}
\hline \hline Action & $\begin{array}{c}\text { Data taken } \\
\text { from }\end{array}$ & Lattice data & $\Lambda_{L}[\mathrm{Mev}]$ & $X_{n, v}$ & $Y_{n^{\prime}, v^{\prime}}$ & $\chi^{2} / d o f$ \\
\hline \hline \multirow{3}{*}{ Wilson } & {$[1]$} & $r_{c}$ & $7.54(4)$ & $.23(2)$ & $-.03(2)$ & .69 \\
& {$[1]$} & $T_{c}$ & $7.53(3)$ & $.213(5)$ & $-.016(2)$ & .87 \\
& {$[4]$} & $\sigma$ & $7.02(9)$ & $.25(2)$ & $-.02(1)$ & .19 \\
\hline \multirow{5}{*}{ Iwasaki } & {$[1,6]$} & $r_{0}$ & $3.62(2)$ & $.046(1)$ & $-.0106(6)$ & .15 \\
& {$[1]$} & $T_{c}$ & $3.68(2)$ & $.121(3)$ & $-.024(1)$ & 14 \\
& {$[6]$} & $\sigma$ & $3.49(4)$ & $.13(1)$ & $-.028(4)$ & .27 \\
& {$[6]$} & $K-K^{*}$ & $4.6(5)$ & $.22(6)$ & $-.07(3)$ & .60 \\
\hline DBW2 & {$[1]$} & $r_{0}$ & $1.24(3)$ & $.064(6)$ & $-.019(4)$ & 4.1 \\
& {$[1]$} & $T_{c}$ & $1.248(4)$ & $.2756(6)$ & - & 3.7 \\
\hline \hline
\end{tabular}

Table 1: Results from the fits of lattice data to lattice distorted PT, using the 3-loop $f_{P T}$ for the Wilson action and the 2-loop $f_{P T}$ for the Iwasaki and DBW2 actions .

being used in the Monte Carlo simulations, and on the quantity being used to set the scale, $a$ (see Table 1). Eg. for $T_{c}$ with the Wilson action, we have $n=2, n^{\prime}=4$, and $v=v^{\prime}=0$.

The physical quantities used to set $a$ in this study are: the string tension $\sigma[4,6]$; the length scale $r_{0}$ and $r_{c}[1,6]$, and the critical temperature $T_{c}$ [1]. The $K, K^{*}$ mass point on the plot of $M_{V}$ versus $M_{P S}^{2}$, where $M_{V}$ and $M_{P S}$ are the mass of the vector and pseudoscalar meson respectively [7], provides the fi fth source of $a^{-1}$ data [6]. As well as the Wilson action, data from the Iwasaki and DBW2 action were also studied.

Where possible we fi t data to next-to-leading-order (i.e. Eq. (2.1)) obtaining the coeffi cients $X_{n, v}$ and $Y_{n^{\prime}, v^{\prime}}$ shown in Table 1. At NNLO the results generally don't change from NLO, so we consider the results in Table 1 as our best fi ts. The only exception to this is the case of $K, K^{*}$ mass 


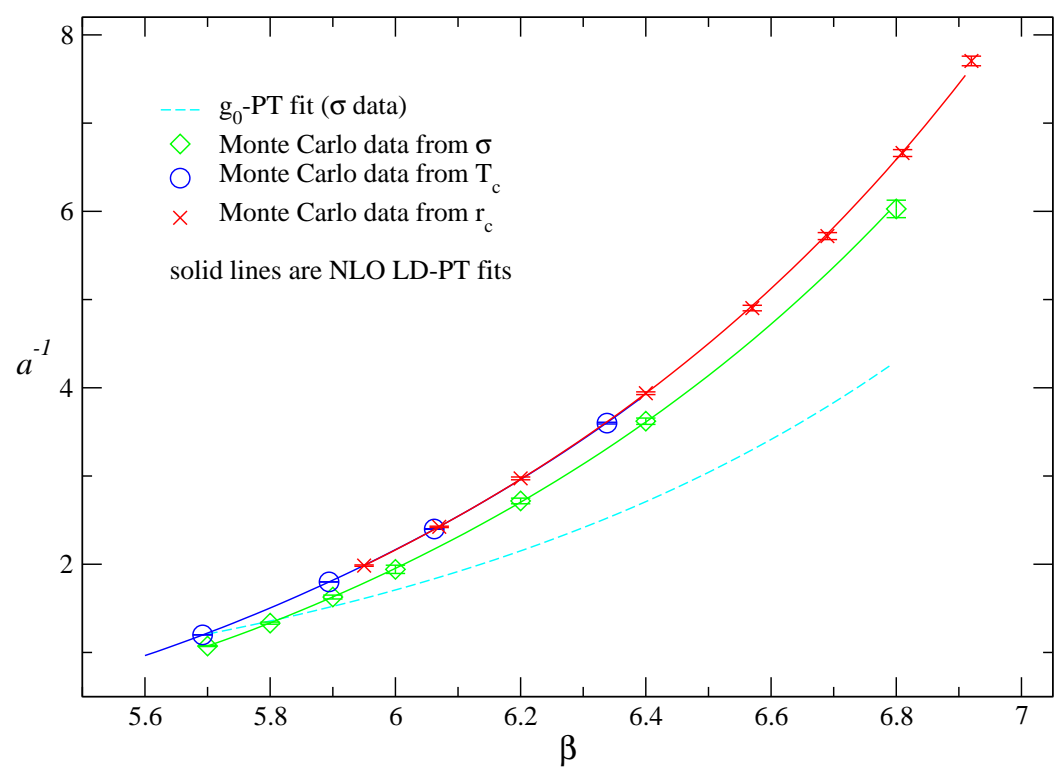

Figure 2: Plot of the Monte Carlo data $a^{-1}$ obtained from observables with the Wilson action together with the NLO lattice-distorted PT curves. The 3-loop $f_{P T}$ function was used.

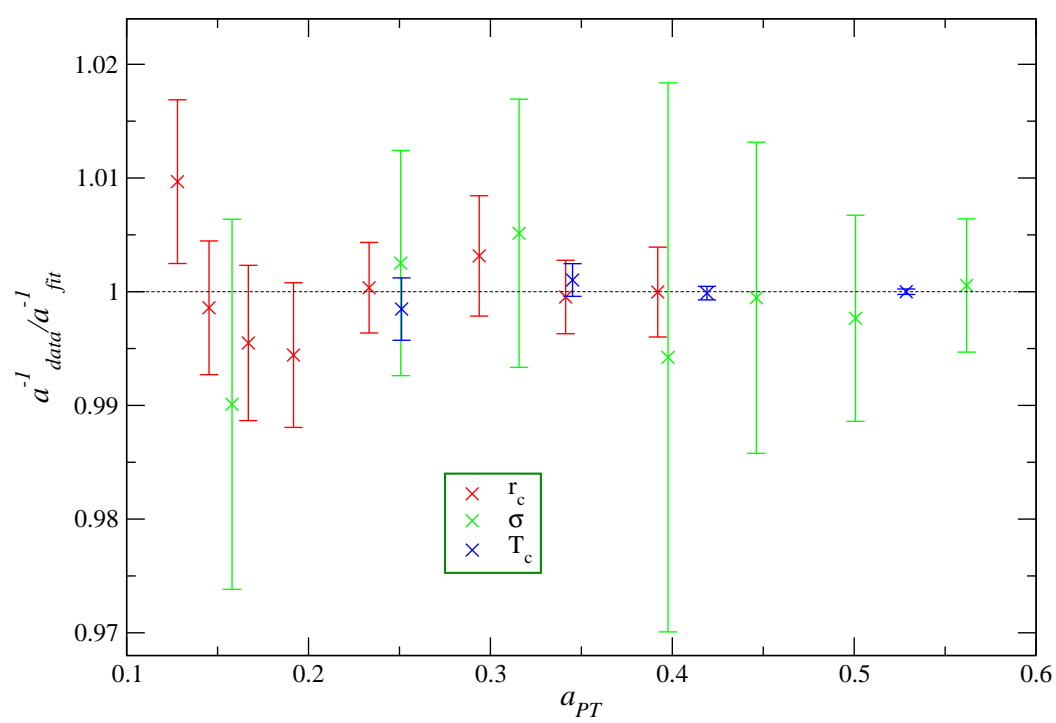

Figure 3: Plot of $a_{\text {data }}^{-1} / a_{f i t}^{-1}$ versus $a_{P T}^{-1}$ for all data in the Wilson case, where $a_{P T}^{-1}=\Lambda_{L} / f_{P T}\left(g_{0}^{2}\right)$. Data from the NLO fit and the 3-loop $f_{P T}$ function were used. 


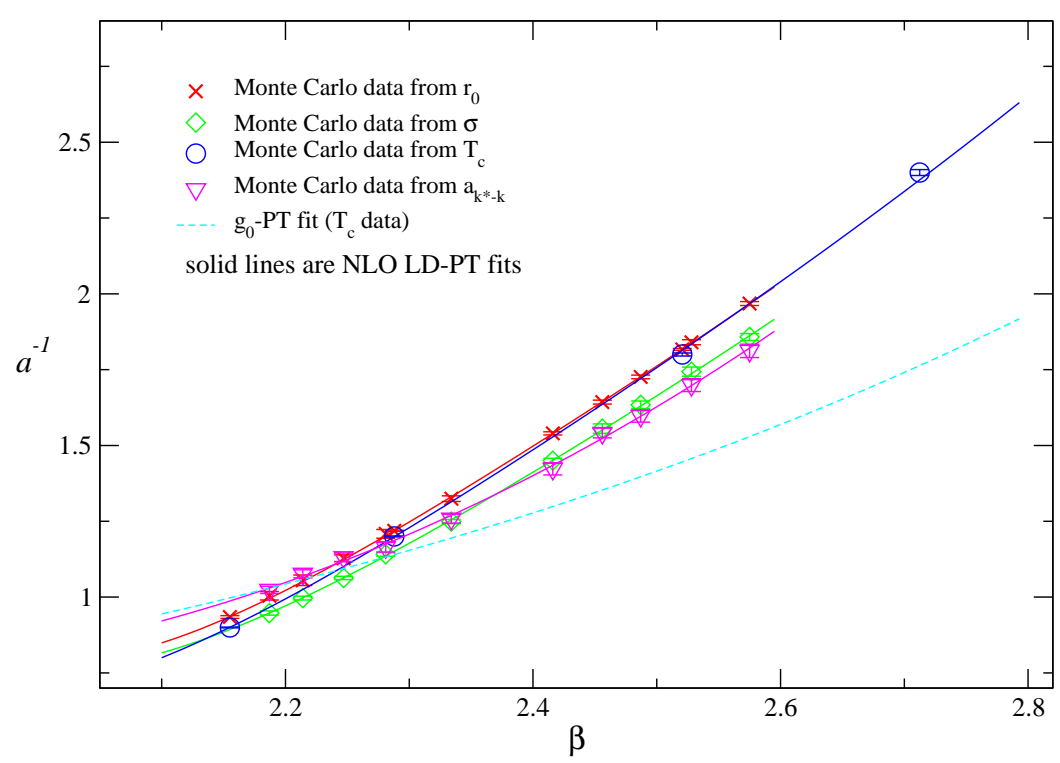

Figure 4: Plot of the Monte Carlo data $a^{-1}$ obtained from observables with the Iwasaki action together with the NLO lattice-distorted PT curves. The 2-loop $f_{P T}$ function was used.

point, where the NNLO fi t fi nds a $\Lambda$ value much closer to that from other quantities. The DBW2 fi $t$ for $T_{c}$ was constrained to be at $\mathrm{LO}$ due to the small number of data points available. The Wilson data was fi tted to 3-loops, whereas the Iwasaki and DBW2 actions to 2-loops.

We plot the data and fi ts for $a^{-1}$ versus $\beta$ in Figures 2 and 4 for the Wilson and Iwasaki actions respectively. In order to show the high level of agreement between the data and lattice distorted perturbation theory, we plot the ratio of the data to the fit in 3 and 5 showing agreement at the percent level.

\section{Conclusions}

Note that the quality of the fi ts (as indicated by the $\chi^{2}$ in Table 1 , and Figs. $3 \& 5$ ) is generally excellent. Furthermore, the $\Lambda_{L}$ value for each action is remarkably consistent (see remarks in Sec. 2 regarding the $K-K^{*}$ fi t). Note also that the series in $a$ appears convergent, in that $Y_{n^{\prime}, v^{\prime}}<<X_{n, v}$ for all cases. Finally, $X_{n, v}$, which is a measure of the leading-order lattice systematics, is $\sim 20 \%$ in the Wilson case, and, as expected, it is signifi cantly smaller for the improved actions. (Note that there are only three $T_{c}$ data points for the DBW2 action, hence the fi t parameters in this case can be discounted.)

Converting $\Lambda_{L}$ for the $r_{c}$ and $T_{c}$ Wilson case to $\Lambda_{\overline{M S}}$ [8], we obtain ${ }^{3}$

$$
\Lambda_{\overline{M S}}^{N_{f}=0}=217 \pm 21 \mathrm{Mev},
$$

in agreement with previous lattice determinations in quenched QCD [5, 4].

Finally, since the lack of perturbative scaling is probably due to a mixture of both lattice artefacts and the poor convergence of the $g_{0}-\mathrm{PT}$, we have started to fi t data to Lattice-Distorted

\footnotetext{
${ }^{3}$ We are still investigating the extraction of $\Lambda_{\overline{M S}}$ from the Iwasaki and DBW2 fi ts.
} 


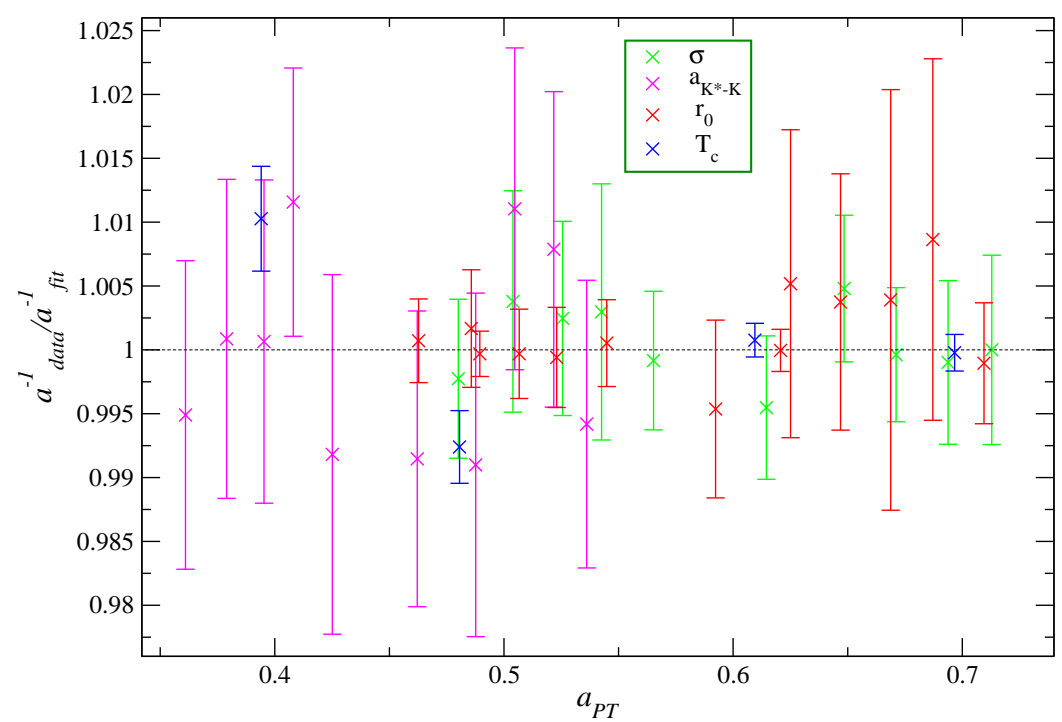

Figure 5: Plot of $a_{\text {data }}^{-1} / a_{f i t}^{-1}$ versus $a_{P T}^{-1}$ for all data in the Iwasaki case, where $a_{P T}^{-1}=\Lambda_{L} / f_{P T}\left(g_{0}^{2}\right)$. Data from the NLO fit and the 2-loop $f_{P T}$ function were used.

PT using a renormalized coupling constant instead of $g_{0}$ (see [9]). In particular, in the $g_{E}$ scheme where the 3-loop coeffi cient is known, we obtain results consistent with [4], but the addition of the $\mathscr{O}\left(a^{n}\right)$ terms improves the quality of the fi t.

\section{References}

[1] S. Necco, Ph.D. Thesis (Humboldt U., Berlin) June 2003, [arXiv: hep-lat/0306005] .

[2] G.P. Lepage and P.B. Mackenzie, Phys. Rev. D48 (1993) 2250, [arXiv: hep-lat/9209022 ].

[3] G. Parisi, LNF-80/52-P Presented at 20th Int. Conf. on High Energy Physics, Madison, Wis., Jul $17-23,1980$

[4] G.S. Bali and K. Schilling, Phys. Rev. D47 (1993) 661, [arXiv: hep-lat/9208028] .

[5] C.R. Allton, [arXiv: hep-lat/9610016]; Nucl. Phys. B(Proc.Suppl.) 53 (1997) 867, [arXiv:hep-lat/9610014]

[6] A. Ali Khan et al. [CP-PACS Collaboration], Phys. Rev. D65 (2002) 054505, [Erratum-ibid. D 67 (2003) 059901], [arXiv: hep-lat/0105015] .

[7] C.R. Allton, V. Gimènez, L. Giusti, and F. Rapuano, Nucl. Phys. B489 (1997) 427 [arXiv : hep-lat/9611021]

[8] R. Dashen and D.J. Gross, Phys. Rev. D23 (1981) 2340.

[9] R.G. Edwards, U.M. Heller, T.R. Klassen, Nucl. Phys. B517 (1998) 377, [arXiv:hep-lat/9711003]. 